\title{
Avaliação da biocompatibilidade de cimentos reparadores biocerâmicos: Estudo in vivo em ratos wistar
}

\author{
Biocompatibility assessment of bioceramic repair cements: An in vivo study in wistar rats \\ Evaluación de la biocompatibilidad de los cementos reparadores biocerámicos: Un estudio in vivo \\ en ratas wistar
}

Recebido: 29/03/2021 | Revisado: 10/04/2021 | Aceito: 28/05/2021 | Publicado: 11/06/2021

Diego Valentim

ORCID: https://orcid.org/0000-0002-5699-6578 Universidade Estadual Paulista, Brasil E-mail: dvalentim_5@hotmail.com

Carlos Roberto Emerenciano Bueno ORCID: https://orcid.org/0000-0002-1897-2823 Universidade Estadual do Norte do Paraná, Brasil E-mail: dentistabueno@gmail.com

Vanessa Abreu Sanches Marques ORCID: https://orcid.org/0000-0003-0509-6956 Universidade Estadual Paulista, Brasil E-mail:van.marqs@gmail.com

Francine Benetti

ORCID: https://orcid.org/0000-0002-5459-353X

Universidade Federal de Minas Gerais, Brasil

E-mail: Francine_Benetti@hotmail.com

Ana Maria Veiga Vasques

ORCID: https://orcid.org/0000-0002-1211-2363 Universidade Estadual Paulista, Brasil

E-mail: anavvasques@hotmail.com

Marina Tolomei Sandoval Cury

ORCID: https://orcid.org/0000-0003-0733-7936 Universidade Estadual Paulista, Brasil E-mail: ma.tolomei@gmail.com

Ana Claudia Rodrigues da Silva

ORCID: https://orcid.org/0000-0002-7570-6126 Universidade Estadual Paulista, Brasil E-mail: claudia.silva@unesp.br

Rogério Castilho Jacinto

ORCID: https://orcid.org/0000-0002-2362-8920 Universidade Estadual Paulista, Brasil E-mail: rogerio.castilho@unesp.br

Gustavo Sivieri-Araújo

ORCID: https://orcid.org/0000-0002-8402-7408 Universidade Estadual Paulista, Brasil E-mail: gustavo.sivieri@unesp.br

Luciano Tavares Angelo Cintra

ORCID: https://orcid.org/0000-0003-2348-7846 Universidade Estadual Paulista, Brasil E-mail: luciano.cintra@unesp.br

Eloi Dezan Junior

ORCID: https://orcid.org/0000-0002-5699-6578 Universidade Estadual Paulista, Brasil E-mail: eloi.dezan@unesp.br

\begin{abstract}
Resumo
O objetivo deste estudo foi avaliar a biocompatibilidade do cimento biocerâmico reparador Biodentine ${ }^{\circledR}$ quando comparado ao MTA Branco Angelus ${ }^{\circledR}$ e hidróxido de cálcio. Para isso foram utilizados vinte e quatro ratos Wistar (n=6 animais/grupo), divididos em quatro tempos experimentais de 7, 15, 30 e 60 dias. Os animais receberam implantes subcutâneos de tubos de polietileno contendo os 3 materiais e um tubo vazio utilizado como controle. Após os períodos experimentais os animais foram eutanasiados e os tubos, juntamente com o tecido circundante, foram removidos e processados histologicamente para avalição da biocompatibilidade. Infiltrado inflamatório e espessura de cápsula fibrosa foram analisados por coloração de HE através de escores de inflamação. Os dados foram submetidos ao teste de Kruskal Wallis e Dunn $(\mathrm{p}<0,05)$. O Biodentine ${ }^{\circledR}$ aos 15 dias gerou menor resposta inflamatória que o controle e o
\end{abstract}


$\mathrm{Ca}(\mathrm{OH})_{2}(\mathrm{p}<0,05)$. No grupo hidróxido de cálcio, apartir dos 15 dias em diante houve diminuição do infiltrado inflamatório, assim como diminuição da cápsula fibrosa. O MTA Branco Angelus ${ }^{\circledR}$ mostrou capsula fibrosa fina e baixa intensidade inflamatório em todos os períodos. Após os períodos experimentais, todos materiais apresentaram inflamação leve e capsula fibrosa fina. Conclui-se que o Biodentine ${ }^{\circledR}$ demonstrou biocompatibilidade tecidual pois induziu baixa inflamação, que reduziu ao longo do tempo, comparável ao MTA Angelus Branco ${ }^{\circledR}$ e hidróxido de cálcio. Palavras-chave: Pulpotomia; Inflamação; Teste de materiais; Endodontia.

\begin{abstract}
The aim of this study was to evaluate the biocompatibility of Biodentine ${ }^{\circledR}$ bioceramic repair cement when compared to White Angelus MTA ${ }^{\circledR}$ and calcium hydroxide. Twenty-four Wistar rats were used ( $\mathrm{n}=6$ animals / group), divided into four experimental periods of 7, 15, 30 and 60 days. The animals received subcutaneous implants of polyethylene tubes containing the 3 test materials and an empty tube used as control. After experimental periods, animals were euthanized and the tubes were removed along with the adjacent surrounding tissue and histologically processed to assess biocompatibility. Inflammatory infiltrate and fibrous capsule thickness were analyzed by HE staining through inflammation scores. Data was submitted to Kruskal Wallis and Dunn's test $(\mathrm{p}<0.05)$. After 15 days, Biodentine ${ }^{\circledR}$ induced less inflammatory response than control group and $\mathrm{Ca}(\mathrm{OH})_{2}(\mathrm{p}<0.05)$. In calcium hydroxide group there was a decrease in the inflammatory infiltrate, as well as a decrease in the fibrous capsule, from day 15 to 60 . The White Angelus MTA $^{\circledR}$ showed thin fibrous capsule and low inflammatory intensity in all periods. At the endo of experiment, all materials showed mild inflammation and thin fibrous capsule. It is concluded that Biodentine ${ }^{\circledR}$ demonstrated tissue biocompatibility as it induced low inflammation, which reduced over time, comparable White Angelus MTA ${ }^{\circledR}$ and calcium hydroxide.
\end{abstract}

Keywords: Pulpotomy; Inflammation; Materials testing; Endodontics.

\title{
Resumen
}

El objetivo de este estudio fue evaluar la biocompatibilidad del cemento biocerámico reparador Biodentine ${ }^{\circledR}$ en comparación con MTA Branco Angelus ${ }^{\circledR}$ e hidróxido de calcio. Para ello se utilizaron veinticuatro ratas Wistar $(n=6$ animales / grupo), divididas en cuatro tiempos experimentales de 7, 15, 30 y 60 días. Los animales recibieron implantes subcutáneos de tubos de polietileno que contenían los 3 materiales y un tubo vacío utilizado como control. Después de los períodos experimentales, los animales fueron sacrificados y los tubos, junto con el tejido circundante, se retiraron y se procesaron histológicamente para evaluar la biocompatibilidad. El infiltrado inflamatorio y el espesor de la cápsula fibrosa se analizaron mediante tinción con HE a través de puntuaciones de inflamación. Los datos se sometieron a la prueba de Kruskal Wallis y Dunn ( $\mathrm{p}<0,05$ ). Biodentine ${ }^{\circledR}$ a los 15 días generó menos respuesta inflamatoria que el control y $\mathrm{Ca}(\mathrm{OH})_{2}$ ( $\left.\mathrm{p}<0.05\right)$. A partir de los días 15,30 y 60 hubo una disminución del infiltrado inflamatorio, así como una disminución de la cápsula fibrosa, en el grupo de hidróxido de calcio. El White Angelus ${ }^{\circledR}$ MTA mostró cápsula fibrosa fina y baja intensidad inflamatoria en todos los períodos. Después de los períodos experimentales, todos los materiales mostraron una leve inflamación y una fina cápsula fibrosa. Se concluye que Biodentine ${ }^{\circledR}$ demostró biocompatibilidad tisular porque indujo una baja inflamación, que se redujo con el tiempo, comparable a MTA Angelus Branco $^{\circledR}$ e hidróxido de calcio.

Palabras clave: Pulpotomía; Inflamación; Ensayo de materiales; Endodoncia.

\section{Introdução}

Quando a exposição da polpa provoca lesões pulpares reversíveis, em dentes em desenvolvimento ou maduros, o capeamento pulpar direto e indireto são formas de preservar a vitalidade pulpar via a formação da ponte de dentina. Durante a formação da dentina, células da polpa dentária sofrem proliferação e diferenciação em células odontoblásticas que secretam proteínas da matriz dentinária que induzem a mineralização da dentina (Tziafas et al.,1995).

Diferentes materiais são utilizados em terapias onde a polpa encontra-se com vitalidade, sendo o prognóstico do tratamento dependente de fatores como a biocompatibilidade e a capacidade de prevenir a infiltração bacteriana, além do resultado também depender da capacidade de resposta da polpa frente à agressão (Camps et al., 2000). O material ideal para ser utilizado na proteção do complexo dentino-pulpar deve ser biocompatível (Minamikawa et al., 2011), ou seja, não provocar injúrias no tecido que está em contato. 
O hidróxido de cálcio $\left[\mathrm{Ca}(\mathrm{OH})_{2}\right]$ é amplamente utilizado quando se trata de agente de proteção pulpar (Paranjpe et al., 2010). No entanto, evidências sugerem que a infiltração e a porosidade podem ocorrer devido a sua elevada solubilidade em água, o que poderia levar ao fracasso do capeamento pulpar (Schuurs et al., 2000; AI-Hezaimi et al., 2011). Além disso, o hidróxido de cálcio destrói uma fina camada do tecido pulpar subjacente, deixando uma camada necrótica, devido ao seu pH elevado (Ford et al., 1995).

$\mathrm{O}$ agregado de trióxido mineral (MTA) pode ser empregado como alternativa ao $\mathrm{Ca}(\mathrm{OH})_{2}$ para tratamento de injúrias pulpares, estimulando a formação de pontes de dentina. O MTA é um material bioativo, biocompatível, estável e com alta capacidade de vedação (Faraco et al., 2001; Eskandarizadeh et al., 2011; Bueno et al., 2019). Entretanto, o MTA é difícil de ser manipulado, seu tempo de presa é longo, possui custos elevados e pode causar pigmentação do elemento dental (Dammaschke et al., 2005).

Outro material utilizado como cimento restaurador/forrador é o Biodentine ${ }^{\circledR}$ (Septodont, Saint Maur des Fossés, France), à base de silicato de cálcio, com propriedades mecânicas semelhantes à dentina, podendo ser utilizado como um substituto da dentina na coroa, e tem seu uso similar ao do MTA no canal radicular (Laurent et al., 2012; Koubi et al., 2013).

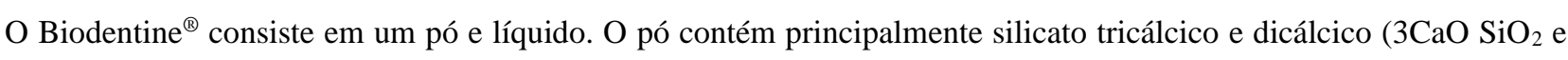
$\left.2 \mathrm{CaO} \mathrm{SiO}_{2}\right)$, o principal componente do cimento Portland, bem como carbonato de cálcio $\left(\mathrm{CaCO}_{3}\right)$ e o dióxido de zircónio $\left(\mathrm{ZrO}_{2}\right)$ serve como meio de contraste. O líquido é composto de cloreto de cálcio $\left(\mathrm{CaCl}_{2} 2 \mathrm{H}_{2} \mathrm{O}\right)$, que é usado como um acelerador de configuração e o agente de redução de água numa solução aquosa com uma mistura de policarboxilato (um agente superplastificante) (Laurent et al., 2012; Chicarelli et al., 2021).

Visando a qualidade e eficácia dos materiais lançados no mercado, o objetivo deste estudo foi avaliar a resposta tecidual do cimento reparador biocerâmico Biodentine ${ }^{\circledR}$, quando comparado ao MTA Branco Angelus ${ }^{\circledR} \mathrm{e} \mathrm{Ca}(\mathrm{OH})_{2}$, por meio da análise histológica em tecido subcutâneo de ratos Wistar.

\section{Material e Métodos}

\section{Animais}

O cálculo amostral foi baseado em um estudo prévio (Chicarelli et al., 2021): considerando um poder de análise de 0.80, diferença mínima entre as médias de 0,22 e desvio padrão (SD) do erro médio de 0.11 , garantiu um mínimo de seis animais (n=6) por grupo experimental.

Vinte e quatro ratos Wistar machos, pesando 250-280g, foram utilizados neste estudo. Os animais foram alojados em gabinetes com temperatura controlada e com água e comida ad libitum. O estudo foi realizado de acordo com as normas da Comissão de Ética da Faculdade de Odontologia de Araçatuba - UNESP, Processo nº 2014-00452.

\section{Implante subcutâneo}

Setenta e dois tubos de polietileno (Abbott Laboratories do Brasil, São Paulo, SP, Brasil), com um diâmetro interno de 1,0 mm, diâmetro externo 1,6 mm, e 10,0 mm de comprimento (Bueno et al., 2019; Benetti et al., 2020) foram preenchidos com um dos materiais experimentais: $\mathrm{Ca}(\mathrm{OH})_{2}+$ água destilada, MTA Angelus Branco $^{\circledR}$, Biodentine ${ }^{\circledR}$ e 24 tubos foram utilizados como controle, portanto, não foram preenchidos.

Para realização da intervenção cirúrgica, os 24 animais divididos em 4 períodos de tempo $(\mathrm{n}=6)$ foram submetidos à anestesia geral com xilazina $(25 \mathrm{mg} / \mathrm{kg})$ e quetamina $(50 \mathrm{mg} / \mathrm{kg})$, via intramuscular. Uma pequena área dorsal foi depilada, a antissepsia foi obtida com solução de iodo a 5\%, foi realizada uma incisão longitudinal com lâmina de bisturi número $15 \mathrm{C}$ acompanhando a linha mediana, com extensão de no máximo $1 \mathrm{~cm}$ atingindo o tecido subcutâneo. Desta forma, com o auxílio de um trocarte adaptado os tubos de polietileno foram implantados: dois do lado direito (superior e inferior) e dois no lado esquerdo 
(superior e inferior) no tecido subcutâneo dos animais. A incisão foi suturada com fio de seda 4-0 (Johnson \& Johnson Produtos Profissionais Ltda, São José dos Campos, Brasil).

Após 7, 15, 30 e 60 da implantação, os animais foram eutanasiados por overdose anestésica e os tubos, com os tecidos circundantes, foram removidos e fixados em solução formalina à 10\% tamponada em pH neutro por 24 horas, seguido de lavagem em água corrente por 12 horas. Os espécimes foram incluídos em parafina para obtenção de cortes seriados de $5 \mu$ m, para coloração em hematoxilina-eosina (HE).

A resposta tecidual produzida pelos materiais foi avaliada conforme estudos anteriores (Cintra et al., 2013., Bueno et al., 2016; Bueno et al., 2019; Benetti et al., 2020): 0 - ausência de células inflamatórias, sem reação; 1 - menos de 25 células, reação leve; 2 - entre 25 e 125 células inflamatórias, reação moderada; e 3 - 125 ou mais células inflamatórias, reação grave (400X). A Cápsula fibrosa foi considerada fina quando menor que $150 \mu \mathrm{m}$ e espessa quando maior que $150 \mu \mathrm{m}$. (100X). Os dados foram analisados estatisticamente pelo teste de Kruskal-Wallis e Dunn. O valor de $\mathrm{p}<0,05$ foi considerado significativo.

\section{Resultados}

\section{Controle}

Aos 7, 15 e 30 dias, o grupo controle apresentou um processo inflamatório moderado, com presença de macrófagos e linfócitos, além de uma cápsula fibrosa espessa (Figura 1 - A, E, I). Aos 60 dias, observou-se a formação de tecido conjuntivo bem organizado, com poucas células inflamatórias (Figura 1 - M).

\section{Biodentine $^{\circledR}$}

Aos 7 dias observou-se infiltrado inflamatório moderado, presença de células gigantes, fibroblastos e vasos sanguíneos (Figura 1- B). Após 15 dias o Biodentine ${ }^{\circledR}$ gerou menor resposta inflamatória que o controle e o $\mathrm{Ca}(\mathrm{OH})_{2}(\mathrm{p}<0,05)(\mathrm{Figura} 1 . \mathrm{F}$, Tabela 1). Nos períodos de 30 e 60 dias houve uma redução no número de células inflamatórias (Figura 1 - J, N). A cápsula fibrosa aos 7 dias (Figura 1 - B) se encontrava espessa e a partir dos 15, 30 e 60 dias foi observado a diminuição da capsula fibrosa (Figura 1 - F, J, N, Tabela 1).

\section{Hidróxido de Cálcio}

No período de 7 e 15 dias houve presença de infiltrado inflamatório moderado, com linfócitos, macrófagos (Figura 1 -C, G). A cápsula fibrosa se mostrou espessa aos 7 dias (Figura 1- C), a partir dos 15, 30 e 60 dias houve a diminuição da cápsula (Figura 1 - G, K, O). Aos 30 e 60 dias, o tecido mostrou reação inflamatória leve, com poucas células inflamatórias (Figura 1 $\mathrm{K}, \mathrm{O})$.

\section{MTA Branco Angelus ${ }^{\circledR}$}

Aos 7, 15, 30 e 60 dias foram observados uma cápsula fibrosa fina, (Figura 1 - D, H, L, P). Aos 7 e 15 dias foi observado moderado número de células inflamatórias identificando a presença de linfócitos, neutrófilos e macrófagos. Aos 30 e 60 dias, foi observado menor intensidade do infiltrado inflamatório. 
Figura 1. (HE - 100X). Imagens representativas da reação inflamatória dos grupos experimentais e grupo controle. Aos 7 dias, os grupos controle (A), Biodentine $(\mathrm{B})$ e $\mathrm{Ca}(\mathrm{OH})_{2}(\mathrm{C})$ apresentaram infiltrado inflamatório moderado e cápsula fibrosa espessa; o grupo MTA Branco Angelus ${ }^{\circledR}$ (D) apresentou infiltrado inflamatório moderado e cápsula fibrosa fina. Aos 15 dias: o grupo controle (E) apresentou infiltrado inflamatório moderado e cápsula fibrosa espessa, o Biodentine $(\mathrm{F}), \mathrm{o} \mathrm{Ca}(\mathrm{OH})_{2}(\mathrm{G})$ e o $\mathrm{MTA}$ $(\mathrm{H})$, apresentaram infiltrado inflamatório moderado e cápsula fibrosa fina. Aos 30 dias, o controle (I) apresentou infiltrado inflamatório moderado e cápsula fibrosa fina. Os grupos Biodentine $(\mathrm{J}), \mathrm{Ca}(\mathrm{OH})_{2}(\mathrm{~K})$ e o MTA (L) apresentaram infiltrado inflamatório leve e cápsula fibrosa fina; Aos 60 dias, todos os 4 grupos (M, N, O, P) apresentaram infiltrado inflamatório leve e capsula fibrosa fina.
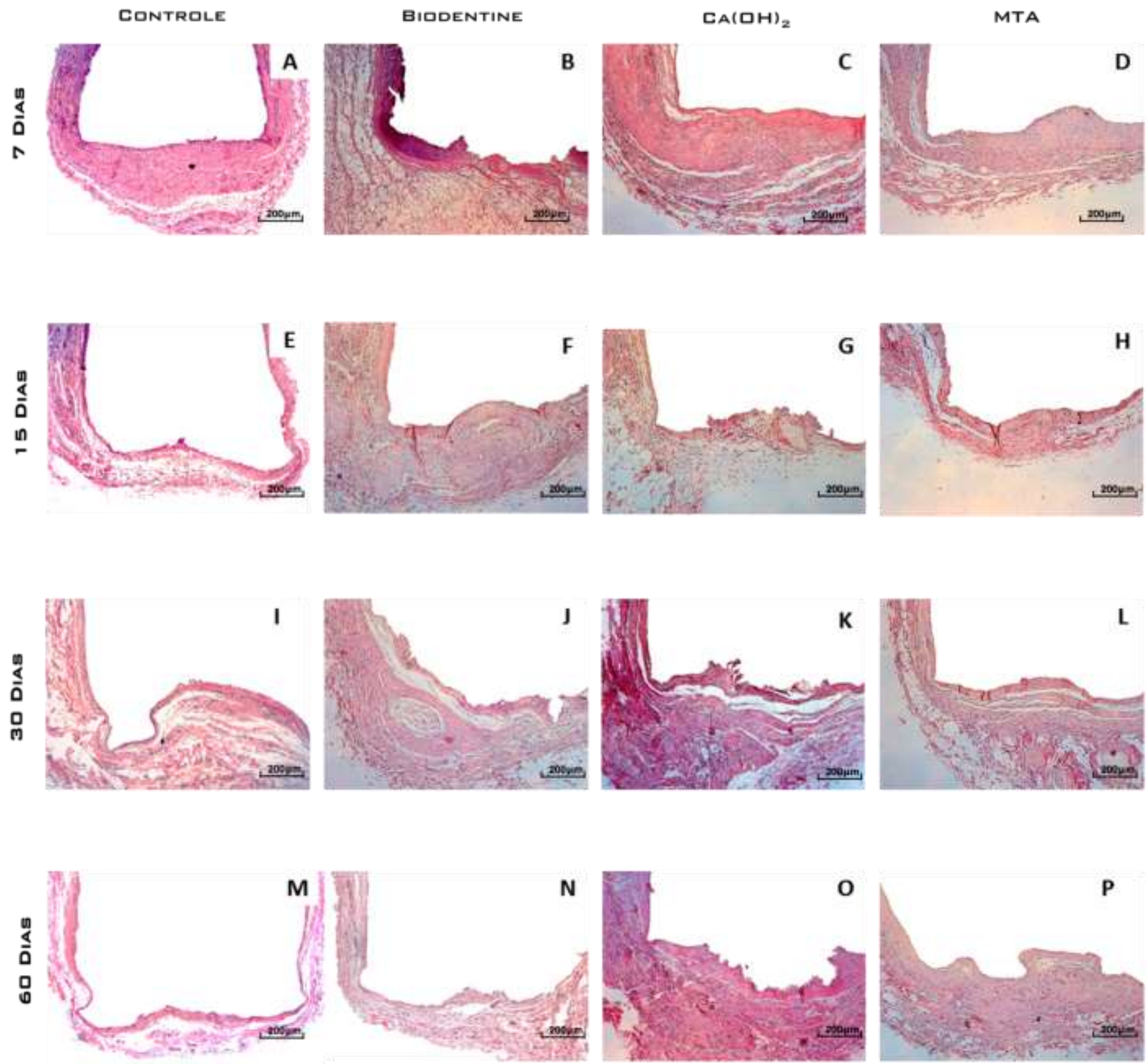

Fonte: Autores. 
Tabela 1. Classificação dos espécimes de acordo com escores para infiltrado inflamatório e espessura da cápsula fibrosa. Letras iguais indicam que não houve diferença estatística entre os grupos ( $\mathrm{p}>5 \%)$.

\begin{tabular}{|c|c|c|c|c|c|c|c|}
\hline \multirow[t]{2}{*}{ Tempo } & \multirow[t]{2}{*}{ Material } & \multicolumn{4}{|c|}{$\begin{array}{l}\text { Escores para Infiltrado } \\
\text { Inflamatório }\end{array}$} & \multirow[t]{2}{*}{ Mediana } & \multirow[t]{2}{*}{ Cápsula Fibrosa } \\
\hline & & 0 & 1 & 2 & 3 & & \\
\hline \multirow[t]{4}{*}{7 dias } & Controle & $0 / 6$ & $0 / 6$ & $6 / 6$ & $0 / 6$ & $1^{\mathrm{a}}$ & Espessa \\
\hline & MTA Branco Angelus & $0 / 6$ & $3 / 6$ & $1 / 6$ & $2 / 6$ & $2^{\mathrm{a}}$ & Fina \\
\hline & Biodentine & $0 / 6$ & $2 / 6$ & $3 / 6$ & $1 / 6$ & $1^{\mathrm{a}}$ & Espessa \\
\hline & $\mathrm{Ca}(\mathrm{OH})_{2}$ & $0 / 6$ & $0 / 6$ & $6 / 6$ & $0 / 6$ & $2^{\mathrm{a}}$ & Espessa \\
\hline \multirow[t]{4}{*}{15 dias } & Controle & $0 / 6$ & $0 / 6$ & $6 / 6$ & $0 / 6$ & $2^{\mathrm{a}}$ & Espessa \\
\hline & MTA Branco Angelus & $0 / 6$ & $1 / 6$ & $5 / 6$ & $0 / 6$ & $2^{\mathrm{ab}}$ & Fina \\
\hline & Biodentine & $0 / 6$ & $2 / 6$ & $4 / 6$ & $0 / 6$ & $1^{b}$ & Fina \\
\hline & $\mathrm{Ca}(\mathrm{OH})_{2}$ & $0 / 6$ & $1 / 6$ & $4 / 6$ & $1 / 6$ & $2^{\mathrm{a}}$ & Fina \\
\hline \multirow[t]{4}{*}{30 dias } & Controle & $0 / 6$ & $5 / 6$ & $1 / 6$ & $0 / 6$ & $2^{\mathrm{a}}$ & Fina \\
\hline & MTA Branco Angelus & $0 / 6$ & $4 / 6$ & $2 / 6$ & $0 / 6$ & $2^{\mathrm{a}}$ & Fina \\
\hline & Biodentine & $1 / 6$ & $4 / 6$ & $1 / 6$ & $0 / 6$ & $1^{\mathrm{a}}$ & Fina \\
\hline & $\mathrm{Ca}(\mathrm{OH})_{2}$ & $0 / 6$ & $5 / 6$ & $1 / 6$ & $0 / 6$ & $2^{\mathrm{a}}$ & Fina \\
\hline \multirow[t]{4}{*}{60 dias } & Controle & $0 / 6$ & $6 / 6$ & $0 / 6$ & $0 / 6$ & $2^{\mathrm{a}}$ & Fina \\
\hline & MTA Branco Angelus & $1 / 6$ & $5 / 6$ & $0 / 6$ & $0 / 6$ & $2^{\mathrm{a}}$ & Fina \\
\hline & Biodentine & $1 / 6$ & $5 / 6$ & $0 / 6$ & $0 / 6$ & $1^{\mathrm{a}}$ & Fina \\
\hline & $\mathrm{Ca}(\mathrm{OH})_{2}$ & $1 / 6$ & $5 / 6$ & $0 / 6$ & $0 / 6$ & $2^{\mathrm{a}}$ & Fina \\
\hline
\end{tabular}

Fonte: Autores.

\section{Discussão}

A proposta deste estudo foi avaliar em subcutâneo de ratos Wistar a resposta tecidual do cimento endodôntico Biodentine ${ }^{\circledR}$ comparado ao MTA Angelus Branco ${ }^{\circledR}$ e hidróxido de cálcio.

Para a metodologia in vivo, o uso de modelos animais como cães (Holland et al., 1999; Holland et al., 2007; DezanJúnior et al., 2021) ou ratos é comumente utilizada (Bueno et al., 2016; Bueno et al., 2018; Bueno et al., 2019; Benetti et al, 2020).

O uso de implantes subcutâneos foi iniciado por Torneck na década de 60 (Torneck, 1966). A pesquisa visava avaliar a reação do tecido conjuntivo subcutâneo de ratos a tubos de polietileno implantados e tornou-se um método preliminar comumente usado para avaliar a biocompatibilidade (Zmener et al., 1990; Gomes-Filho et al., 2012; Cintra et al., 2013; Bueno et al., 2016; Bueno et al., 2018; Bueno et al., 2019; Benetti et al., 2020), estabelecido como metodologia padrão recomendada pela ISO 10993 (ISO 2016). 
No presente estudo, as reações a tubos vazios no grupo controle foram semelhantes aos que foram relatados anteriormente nos artigos supracitados, corroborando resultados previamente publicados.

O MTA Angelus Branco ${ }^{\circledR}$, assim como o hidróxido de cálcio foram utilizados como controle positivo para biocompatibilidade de materiais reparadores, uma vez que estes são consagrados na literatura (Holland et al., 2001; Holland et al., 2002; Estrela \& Holland, 2003; Bernabé et al., 2007; Valentim et al., 2017, Bueno et al., 2019). Após 15 e 30 dias observouse redução da inflamação e capsula fibrosa, em acordo com os resultados observados na literatura.

Quanto ao o Biodentine ${ }^{\circledR}$, nossos resultados mostraram que ao ser implantado no tecido subcutâneo induziu resposta tecidual com menor inflamação em relação ao hidróxido de cálcio $(\mathrm{p}<0,05)$. Em relação ao MTA Angelus Branco ${ }^{\circledR}$ não houve diferença estatística em nenhum dos períodos avaliados, resultado que diverge de um estudo prévio (Mori et al., 2014), que relatou que aos 7 dias o Biodentine ${ }^{\circledR}$ gerou maior inflamação que o MTA. Entretanto aos 15 e 30 dias os resultados são semelhantes, não havendo diferença entre os grupos MTA e Biodentine ${ }^{\circledR}$.

A inflamação inicial moderada aos 7 dias é esperada mesmo em grupo controle, uma vez que existe o trauma cirúrgico, sendo a reação normal do tecido diante de um implante subcutâneo. É também comum observar uma reação inflamatória inicial com o uso de cimentos do tipo MTA devido à alcalinidade. Porém, é importante ressaltar que a biocompatibilidade de um material é alcançada se a reação inflamatória inicial reduzir ao longo do tempo, a um nível não significativo (Gomes-Filho et al., 2012; Bueno et al. 2019).

Avaliando o uso do Biodentine ${ }^{\circledR}$ na formação de tecido duro em polpas dentárias, Kim et al. (2016) compararam a resposta tecidual à pulpotomia em ratos com ProRoot MTA e BioAggregate. Através de micro tomografia (microCT) e análise imunohistoquimica, os autores observaram significativa formação de ponte de tecido mineralizado no grupo Biodentine ${ }^{\circledR}$.

De Rossi et al., em 2014 mostraram que radiograficamente o Biodentine ${ }^{\circledR}$ promoveu maior mineralização em dentes de cães que o MTA, mas quando realizada a análise histológica, ambos materiais tiveram comportamento semelhantes. Tal resultado corrobora com os achados do nosso estudo, pois na análise histológica, o Biodentine ${ }^{\circledR}$ não mostrou diferença significante em relação ao MTA (Simsek et al., 2015; Nowicka et al., 2015).

Diante das indicações de selamento de perfuração do Biodentine, sua resistência de união push-out foi avaliada por Guneser et al., (2013), comparada com ProRoot MTA, amálgama, Dyract AP e IRM. O Biodentine apresentou maior resistência de união push-out frente a vários irrigantes utilizados em endodontia (hipoclorito, clorexidina e solução salina) em comparação ao MTA.

Outro recente estudo em acordo com nossos resultados acerca de biocompatibilidade do MTA Angelus Branco ${ }^{\circledR} \mathrm{e}$ Biodentine $^{\circledR}$ foi demonstrado por Alazrag et al. (2020), no qual evidenciaram a biocompatibilidade, solubilidade e adaptação marginal do MTA Angelus Branco ${ }^{\circledR}$ e Biodentine ${ }^{\circledR}$, indicando estes como material reparador de furca, através de estudo em dentes de cães analisados em microscopia eletrônica de varredura (MEV).

Quanto `formação de uma cápsula fibrosa inicialmente espessa que se torna fina ao longo do experimento, é interessante destacar que, em pesquisas in vivo com tecido conjuntivo, é esperada a formação de uma capsula fibrosa ao redor de tubos implantados, uma vez que faz parte do processo de reparo e indicando a tolerância pelos tecidos (Yaltirik et al., 2004; Sumer et al., 2006; Bueno et al., 2018; Bueno et al., 2019; Benetti et al., 2020).

Em um estudo imunohistoquímico inflamatório do Biodentine, um maior número de marcação de interleucina inflamatória (IL-6) foi observado em comparação ao MTA aos 7 e 15 dias, com uma redução desta imunoexpressão após os 60 dias, sugerindo a biocompatibilidade do Biodentine (da Fonseca et al., 2016). Nossos achados também indicaram uma reposta inflamatória mais acentuada nos períodos iniciais em comparação ao MTA Angelus Branco ${ }^{\circledR}$, que reduziu ao longo do experimento. 
No presente estudo, o Biodentine ${ }^{\circledR}$ mostrou melhor resposta tecidual que o grupo hidróxido de cálcio. É importante ressaltar que a condição sistêmica pode influenciar na resposta tecidual frente aos materiais. Cosme-Silva et al. (2019) demonstraram através de uma pesquisa em subcutâneo de ratos hipertensos, que a a condição sistêmica de hipertensão pode aumentar a resposta inflamatória e reduzir a biomineralização de materiais como MTA e Biodentine ${ }^{\circledR}$. Tal achado evidencia a importância de considerar a saúde do paciente para cada caso individualmente e que condições sistêmicas influenciam o tratamento dentário, não devendo de forma alguma ser considerado à parte.

\section{Conclusão}

Diante dos resultados, os autores consideram que o Biodentine ${ }^{\circledR}$ é biocompativel tanto em períodos iniciais quanto ao longo de 60 dias, e poderá ser utilizado como uma alternativa ao MTA Angelus Branco ${ }^{\circledR} \mathrm{e}$ ao hidróxido de cálcio, quando em contato com tecido vivo. Estudos clínicos e randomizados e proservação a longo prazo são necessários para confirmar achados sobre estes materiais.

\section{Agradecimentos}

Este estudo teve apoio financeiro FAPESP, Processo 2014/02327-0.

\section{Referências}

AI-Hezaimi, K., Al-Tayar, B. A., Bajuaifer, Y. S., Salameh, Z., Al-Fouzan, K., \& Tay, F. R. (2011). A hybrid approach to direct pulp capping by using emdogain with a capping material. Journal of endodontics, 37(5), 667-672.

Alazrag, M. A., Abu-Seida, A. M., El-Batouty, K. M., \& El Ashry, S. H. (2020). Marginal adaptation, solubility and biocompatibility of TheraCal LC compared with MTA-angelus and biodentine as a furcation perforation repair material. BMC oral health, 20(1), 298.

Benetti, F., Bueno, C. R. E., Reis-Prado, A. H., et al. (2020). Biocompatibility, Biomineralization, and Maturation of Collagen by RTR ${ }^{\circledR}$, Bioglass and DM Bone ${ }^{\circledR}$ Materials. Brazilian Dental Journal, 31(5), 477-484.

Bernabé, P. F. E., Gomes-Filho, J. E., Rocha, W. C., Nery, M. J., Otoboni-Filho, J. A., \& Dezan-Júnior, E. (2007). Histological evaluation of MTA as a rootend filling material. International Endodontic Journal, 40(10), 758-65.

Bueno, C. R. E, Valentim, D., Jardim-Junior, E. G., Mancuso, D. N., et al. (2018). Tissue reaction to Aroeira (Myracrodruon urundeuva) extracts associated with microorganisms: an in vivo study. Brazilian Oral Research, 32, e42.

Bueno, C. R. E., Valentim, D., Marques, V. A. S., Gomes-Filho, J. E., Cintra, L. T. A., Jacinto, R. C., \& Dezan-Junior, E. (2016). Biocompatibility and biomineralization assessment of bioceramic-, epoxy-, and calcium hydroxide-based sealers. Brazilian oral research, $30(1)$, S1806-83242016000100267.

Bueno, C. R. E., Vasques, A. M. V., Cury, M. T. S., Sivieri-Araújo, G., Jacinto, R. C., Gomes-Filho, J. E., Cintra, L. T. A., \& Dezan-Júnior, E. (2019). Biocompatibility and biomineralization assessment of mineral trioxide aggregate flow. Clinical Oral Investigation, 23(1),169-177.

Camps, J., Déjou, J., Rémusat, M., \& About, I. (2000). Factors influencing pulpal response to cavity restorations. Dental materials: official publication of the Academy of Dental Materials, 16(6), 432-440.

Chicarelli, L., Webber, M., Amorim, J., Rangel, A., Camilotti, V., Sinhoreti, M., \& Mendonça, M. J. (2021). Effect of Tricalcium Silicate on Direct Pulp Capping: Experimental Study in Rats. European journal of dentistry, 15(1), 101-108.

Cintra, L. T., Ribeiro, T. A., Gomes-Filho, J. E., Bernabé, P. F., Watanabe, S., Facundo, A. C., Samuel, R. O., \& Dezan-Junior, E. (2013). Biocompatibility and biomineralization assessment of a new root canal sealer and root-end filling material. Dental traumatology, 29(2), 145-150.

Cosme-Silva, L., Dal-Fabbro, R., Gonçalves, L. O., Prado, A., Plazza, F. A., Viola, N. V., Cintra, L., \& Gomes Filho, J. E. (2019). Hypertension affects the biocompatibility and biomineralization of MTA, High-plasticity MTA, and Biodentine ${ }^{\circledR}$. Brazilian Oral Research, 33 , e060.

Da Fonseca, T. S., da Silva, G. F., Tanomaru-Filho, M., Sasso-Cerri, E., Guerreiro-Tanomaru, J. M., \& Cerri, P. S. (2016). In vivo evaluation of the inflammatory response and IL-6 immunoexpression promoted by Biodentine and MTA Angelus. International Endodontics Journal, 49(2), 145-53.

Dammaschke, T., Gerth, H. U., Züchner, H., \& Schäfer, E. (2005). Chemical and physical surface and bulk material characterization of white ProRoot MTA and two Portland cements. Dental materials: official publication of the Academy of Dental Materials, 21(8), 731-738.

De Rossi, A., Silva, L. A., Gatón-Hernández, P., Sousa-Neto, M. D., Nelson-Filho, P., Silva, R. A., \& de Queiroz, A. M. (2014). Comparison of pulpal responses to pulpotomy and pulp capping with biodentine and mineral trioxide aggregate in dogs. Journal of endodontics, 40(9), $1362-1369$. 
Dezan-Júnior, E., Bueno, C. R. E., Vasques, A. M. V., De Souza, V., Nery, M. J., Otoboni Filho, J. A., Bernabé, P. F. E., Gomes-Filho, J. E., Cintra, L. T. A., Jacinto, R. C., Sivieri-Araújo, G., \& Holland, R. (2021) Influence of different obturation techniques in coronal bacterial infiltration: study in dogs. Research, Society and Development, 10(4), P. E11010413884.

Eskandarizadeh, A., Shahpasandzadeh, M. H., Shahpasandzadeh, M., Torabi, M., \& Parirokh, M. (2011). A comparative study on dental pulp response to calcium hydroxide, white and grey mineral trioxide aggregate as pulp capping agents. Journal of conservative dentistry: JCD, 14(4), 351-355.

Estrela, C., Holland, R. (2003). Calcium hydroxide: study based on scientific evidences. Journal of Applied Oral Science, 11(4), 269-82.

Faraco, I. M., Jr, \& Holland, R. (2001). Response of the pulp of dogs to capping with mineral trioxide aggregate or a calcium hydroxide cement. Dental traumatology: official publication of International Association for Dental Traumatology, 17(4), 163-166.

Ford, T. R., Torabinejad, M., McKendry, D. J., Hong, C. U., \& Kariyawasam, S. P. (1995). Use of mineral trioxide aggregate for repair of furcal perforations. Oral surgery, oral medicine, oral pathology, oral radiology, and endodontics, 79(6), 756-763.

Gomes-Filho, J. E., Watanabe, S., Lodi, C. S., Cintra, L. T., Nery, M. J., Filho, J. A., Dezan, E., Jr, \& Bernabé, P. F. (2012). Rat tissue reaction to MTA FILLAPEX®. Dental traumatology: official publication of International Association for Dental Traumatology, 28(6), 452-456.

Guneser, M. B., Akbulut, M. B., \& Eldeniz, A. U. (2013). Effect of various endodontic irrigants on the push-out bond strength of biodentine and conventional root perforation repair materials. Journal of Endodontics, 39(3), 380-4.

Holland, R., de Souza, V., Nery, M. J., Otoboni Filho, J. A., Bernabé, P. F., \& Dezan Júnior, E. (1999). Reaction of dogs' teeth to root canal filling with mineral trioxide aggregate or a glass ionomer sealer. Journal of Endodontic, 25(11), 728-30.

Holland, R., Filho, J.A., de Souza, V., Nery, M. J., Bernabé, P. F., \& Dezan-Junior, E. (2001). Mineral trioxide aggregate repair of lateral root perforations. Journal of Endodontics, 27(4), 281-4.

Holland, R., de Souza, V., Nery, M. J., Bernabé, P. F. E., Filho J. A., Dezan-Junior, E., \& Murata, S.S. (2002). Calcium salts deposition in rat connective tissue after the implantation of calcium hydroxide-containing sealers. Journal of Endodontics, 28(3),173-6.

Holland, R., Mazuqueli, L., de Souza, V., Murata, S. S., Dezan Júnior, E., \& Suzuki, P. (2007). Influence of the type of vehicle and limit of obturation on apical and periapical tissue response in dogs' teeth after root canal filling with mineral trioxide aggregate. Journal of Endodontic, 33(6), 693-7.

ISO (2016). International Organization for Standardization. ISO 10993-6: Biological Evaluation of Medical Devices Part 6: Testes for Local Effects after Implantation. Geneva:ISO;2016.

Kim, J., Song, Y. S., Min, K. S., Kim, S. H., Koh, J. T., Lee, B. N., Chang, H. S., Hwang, I. N., Oh, W. M., \& Hwang, Y. C. (2016). Evaluation of reparative dentin formation of ProRoot MTA, Biodentine and BioAggregate using micro-CT and immunohistochemistry. Restorative Dentistry and Endodontics, 41(1), 29-36.

Koubi, G., Colon, P., Franquin, J. C., Hartmann, A., Richard, G., Faure, M. O., \& Lambert, G. (2013). Clinical evaluation of the performance and safety of a new dentine substitute, Biodentine, in the restoration of posterior teeth - a prospective study. Clinical oral investigations, 17(1), 243-249.

Laurent, P., Camps, J., \& About, I. (2012). Biodentine(TM) induces TGF- $\beta 1$ release from human pulp cells and early dental pulp mineralization. International endodontic journal, $45(5), 439-448$.

Minamikawa, H., Yamada, M., Deyama, Y., Suzuki, K., Kaga, M., Yawaka, Y., \& Ogawa, T. (2011). Effect of N-acetylcysteine on rat dental pulp cells cultured on mineral trioxide aggregate. Journal of endodontics, 37(5), 637-641.

Mori, G. G., Teixeira, L. M., de Oliveira, D. L., Jacomini, L. M., \& da Silva, S. R. (2014). Biocompatibility evaluation of biodentine in subcutaneous tissue of rats. Journal of endodontics, 40(9), 1485-1488.

Nowicka, A., Wilk, G., Lipski, M., Kołecki, J., \& Buczkowska-Radlińska, J. (2015). Tomographic Evaluation of Reparative Dentin Formation after Direct Pulp Capping with $\mathrm{Ca}(\mathrm{OH}) 2$, MTA, Biodentine, and Dentin Bonding System in Human Teeth. Journal of endodontics, 41(8), 1234-1240.

Paranjpe, A., Zhang, H., \& Johnson, J. D. (2010). Effects of mineral trioxide aggregate on human dental pulp cells after pulp-capping procedures. Journal of endodontics, 36(6), 1042-1047.

Schuurs, A. H. B., Gruythuysen, R. J. M., \& Wesselink, P. R. (2000). Pulp capping with adhesive resinbased composite versus calcium hydroxide: a review. Endodontics \& dental traumatology,16, 240-250.

Simsek, N., Alan, H., Ahmetoglu, F., Taslidere, E., Bulut, E. T., \& Keles, A. (2015). Assessment of the biocompatibility of mineral trioxide aggregate, bioaggregate, and biodentine in the subcutaneous tissue of rats. Nigerian journal of clinical practice, 18(6), 739-743.

Sumer, M., Muglali, M., Bodrumlu, E., \& Guvenc, T. (2006). Reactions of connective tissue to amalgam, intermediate restorative material, mineral trioxide aggregate, and mineral trioxide aggregate mixed with chlorhexidine. Journal of Endodontics, 32(11), 1094-6.

Torneck, C. D. (1996). Reaction of rat connective tissue to polyethylene tube implants part I. Oral Surgery, Oral Medicine, Oral Pathology, and Oral Radiology, 21(3), 379-87.

Tziafas, D., Alvanou, A., Panagiotakopoulos, N., Smith, A. J., Lesot, H., Komnenou, A., \& Ruch, J. V. (1995). Induction of odontoblast-like cell differentiation in dog dental pulps after in vivo implantation of dentine matrix components. Archives of oral biology, 40(10), 883-893.

Valentim, D., Bueno, C. R. E., Marques, V. A. S., Vasques, A. M. V., Cury, M. T. S., Cintra, L. T. A., \& Dezan-Junior, E. (2017). Calcium hydroxide associated with a new vehicle: Psidium cattleianum leaf extracts. Tissue response evaluation. Brazilian Oral Research, 3, 31:e43. 
Research, Society and Development, v. 10, n. 7, e1610714422, 2021

(CC BY 4.0) | ISSN 2525-3409 | DOI: http://dx.doi.org/10.33448/rsd-v10i7.14422

Yaltirik, M., Ozbas, H., Bilgic, B., \& Issever, H. (2004). Reactions of connective tissue to mineral trioxide aggregate and amalgam. Journal of Endodontics, 30(2), 95-9.

Zmener, O., Guglielmotti, M. B., \& Cabrini, R. L. (1990). Tissue response to an experimental calcium hydroxide-based endodontic sealer: a quantitative study in subcutaneous connective tissue of the rat. Endodontics and dental traumatology, 6(2), 66-72. 